\title{
Palladium-Catalysed Intermolecular 1,2-Diamination of Alkenes
}

\author{
Alvaro Iglesias ${ }^{1}$, Edwin G. Pérez ${ }^{1,2}$, Jonathan Kirsch ${ }^{1}, K^{\prime}$ ilian Muñiz ${ }^{1 *}$ \\ ${ }^{1}$ Institute of Chemical Research of Catalonia (ICIQ), Tarragona, Spain. \\ ${ }^{2}$ Department of Organic Chemistry, Faculty of Chemistry, Pontificia Universidad Católica de Chile, Vicuña \\ Mackenna 4860, Macul, Santiago, Chile
}

Keywords: palladium, alkenes, diamination.

\section{kmuniz@icia.es}

\section{INTRODUCTION}

We have recently been interested in the application of palladium catalysts to realise unprecedented 1,2diamination reactions of alkenes. ${ }^{1-3}$ Within this context, the application of suitable high oxidation state palladium catalysis $^{4}$ represents a key methodology.

\section{RESULTS AND DISCUSSION}

We now report two first protocols for palladium catalysed intermolecular diamination reactions of alkenes, which employ readily available nitrogen sources. ${ }^{5,6}$ The diamination products are formed with complete regioselectivity and chemoselectivity.

Subsequent transformation of these products into more elaborate diamine building blocks will also be discussed.

intermolecular diamination of nonfunctionalised alkenes:

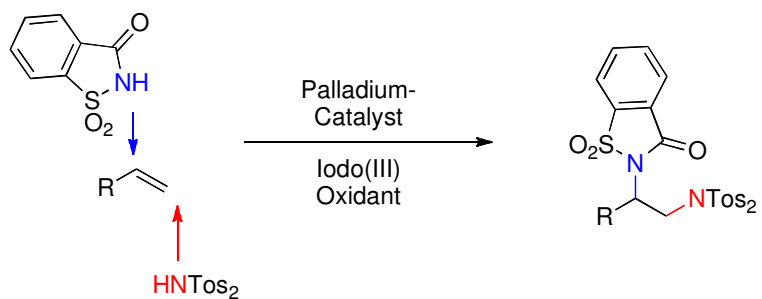

intermolecular diamination of allylic ethers:
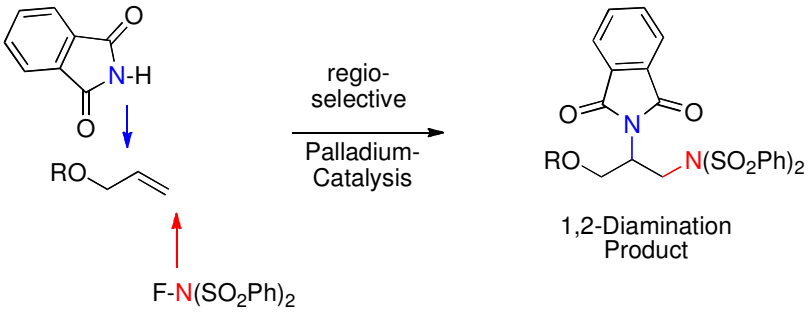

Scheme 1. Intermolecular diamination methods
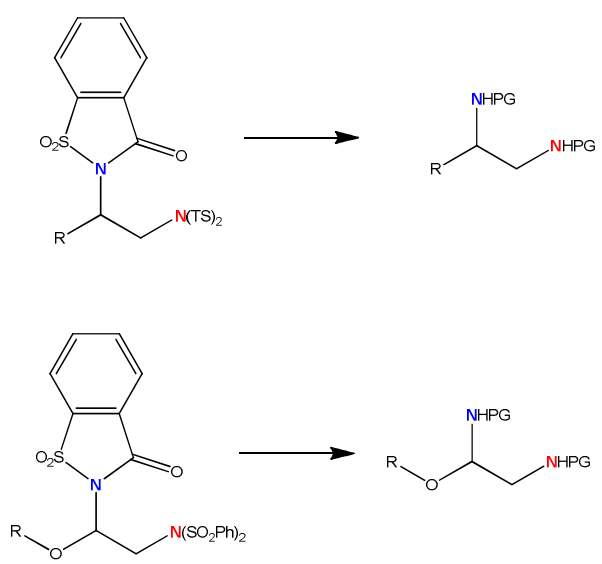

Figure 1. Deprotection of the diamination products to produce new building blocks.

\section{CONCLUSION}

The development of the first palladiumcatalyzed intermolecular diamination of non-activated terminal alkenes. The reaction employs two commercially available nitrogen sources and proceeds with complete regioselectivity under very mild conditions.

\section{ACKNOWLEDGEMENTS}

We thank Fundació ICIQ, the Consolider INTECAT 2010 (Project CSD2006-0003), and the Fonds der Chemischen Industrie for financial support.

E.G.P. was funded by FONDECYT grant 11090120 and ICM grant P05-001-F.

\section{REFERENCES}

(1) K. Muñiz, C. H. Hövelmann, J. Streuff, J. Am. Chem. Soc. 2008, 130, 763.

(2) J. Streuff, C. H. Hövelmann, M. Nieger, K. Muñiz, J. Am. Chem. Soc. 2005, 125, 14586.

(3) K. Muñiz, J. Am. Chem. Soc. 2007, 129, 14542.

(4) K. Muñiz, Angew. Chem. Int. Ed. 2009, 48, 9412.

(5) A. Iglesias, E. G. Pérez, K. Muñiz, Angew. Chem. Int. Ed. 2010, 49, 8109.

(6) J. Kirsch, P. Chávez, K. Muñiz, Adv. Synth. Catal. 2011, 353, 689 . 